\title{
Particle Production for a Muon Storage Ring: II. Radiation Loads *
}

\author{
N.V. Mokhov \\ Fermilab, P.O. Box 500, Batavia, IL 60510, USA ${ }^{1}$
}

\begin{abstract}
Efficient production and collection of a large number of muons is needed to make a neutrino factory based on a muon storage ring viable. Results of extensive MARS simulations are reported for 2 to $30 \mathrm{GeV}$ protons on various targets in a $20 \mathrm{~T}$ hybrid solenoid, followed by a matching section and decay channel. Part II describes radiation loads on targets, capturing system and shielding.
\end{abstract}

Key words: , Muon storage ring, targets, superconducting solenoid, radiation fields PACS: 41.75.-i, 85.25.Ly, 87.18.Bb, 87.53.Wz

\section{Simulations}

The system described in Part I, will provide a muon beam needed for a neutrino factory [1]. A megawatt proton beam hits a thick tilted graphite or mercury target sitting in a high-field superconducting (SC) solenoid. Hadronic and electromagnetic showers are induced in the target and capturing system, resulting in enormous particle fluxes in system components (Fig. 1) which deteriorate their performance rapidly. The SC coils are to be adequately protected to provide their short and long term operation. Full MARS $[2,3]$ simulations have been performed to get accumulated dose and particle fluxes in the system in the high-field, transition and low-field regions, to find an adequate radiation shield, to get residual dose rates on the system components and to estimate ground water and personnel radiation shielding. Results below are for a $1.5 \mathrm{MW} 16 \mathrm{GeV}$ proton beam on a graphite target $(80 \mathrm{~cm}$ long, $0.75 \mathrm{~cm}$ radius, tilted by $50 \mathrm{mrad})$. Preliminary results are in Ref. [1,4].

\footnotetext{
* Work supported by the Universities Research Association, Inc., under contract DE-AC02-76CH00300 with the U. S. Department of Energy.

1 E-mail: mokhov@fnal.gov
} 

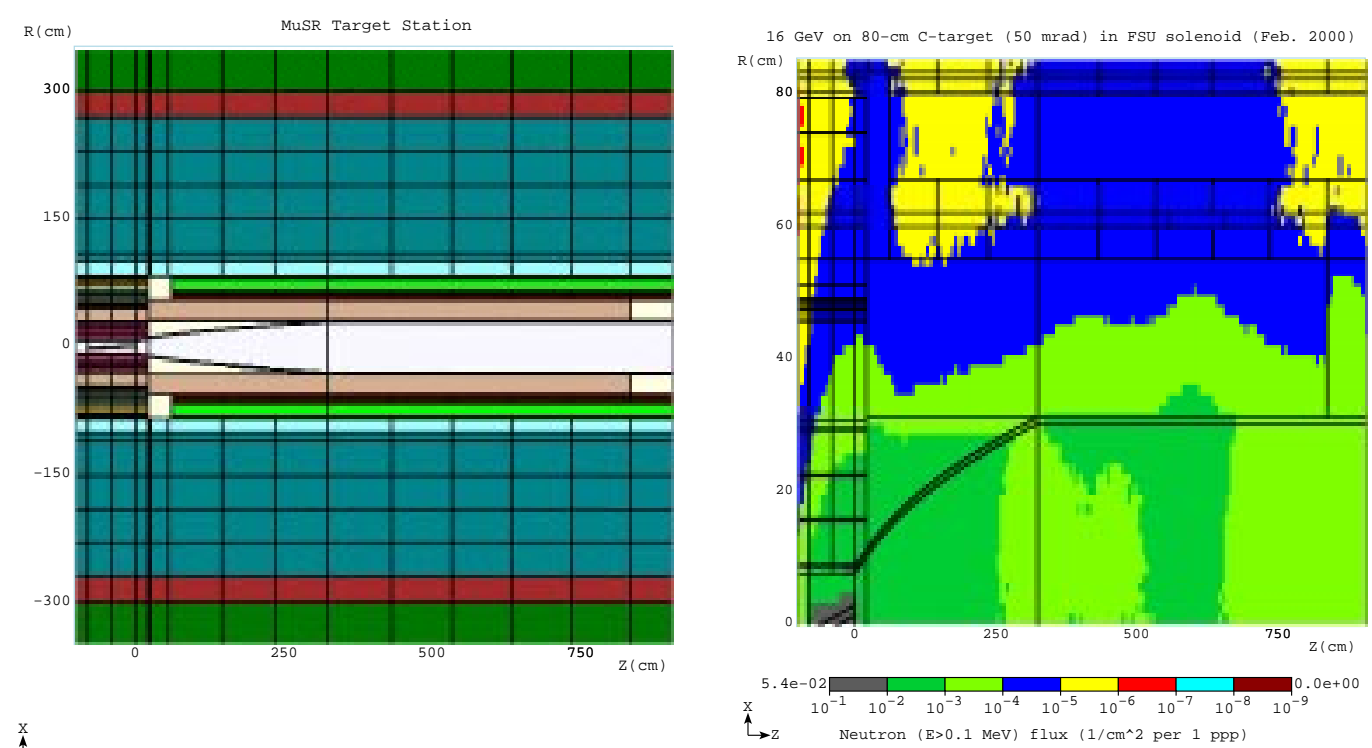

$\stackrel{x}{\leftrightarrow \rightarrow 2}$

Fig. 1. MARS model of the target/solenoid system (left) and isofluxes of neutrons $(\mathrm{E}>0.1 \mathrm{MeV})$ in the system (right).

\section{Particle flux and dose}

There are two pronounced peaks in dose and flux distributions: one at the downstream end of the target at the transition from the 20 - $\mathrm{T}$ region to a matching section, and another one at a primary beam dump at $z \approx 6 \mathrm{~m}$ (Fig. 1). Neutron and photon spectra entering the inner resistive coil and the 20-T SC coil at the first peak are shown in Fig. 2. The spectra span many decades.
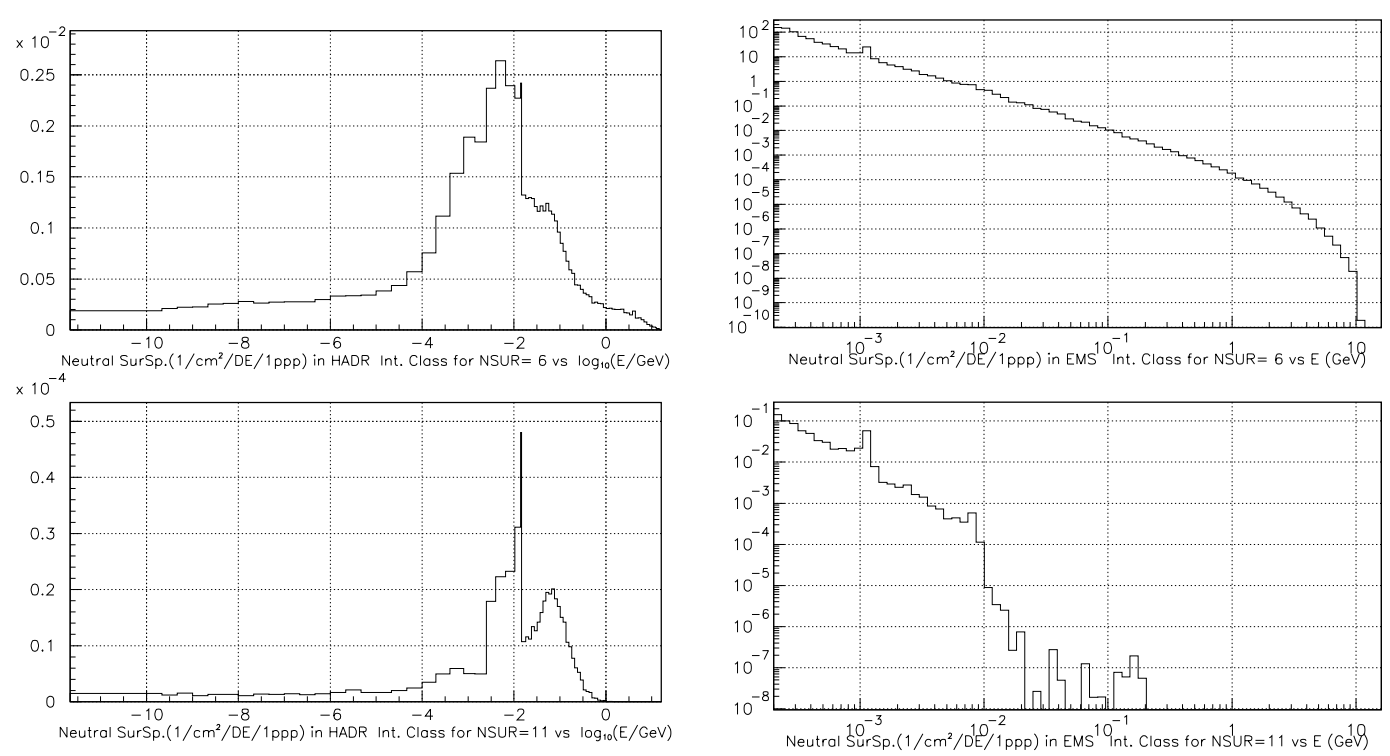

Fig. 2. Neutron $E d N / d E$ (left) and photon $d N / d E$ (right) spectra entering the inner resistive coil at $\mathrm{R}=9 \mathrm{~cm}$ (top) and the $\mathrm{SC}$ windings at $\mathrm{R}=49 \mathrm{~cm}$ (bottom) at $-40<\mathrm{z}<20 \mathrm{~cm}$. 
Thick shielding made of tungsten-carbide balls ( $80 \%$ filling factor) cooled by circulating water is placed between the resistive and SC coils in the 20-T field region, and in front of the potted 1.25- T SC coils in a 3-m long matching section and further in the decay channel. The calculations show that it does an excellent job in protecting the SC coils against radiation. Fig. 3 shows the calculated radial distribution of particle flux, power density and absorbed dose. Prompt dose distribution in the entire system coincides with the energy deposition isocontours shown in Fig. 4 (left). The annual hadron flux in a stationary graphite target is $\sim 5 \times 10^{21} \mathrm{~cm}^{-2}$ which corresponds to several month lifetime. The annual hadron flux $(\mathrm{E}>0.1 \mathrm{MeV})$ and dose in the hottest spot of the inner resistive coil are $1.2 \times 10^{20} \mathrm{~cm}^{-2}$ and $3 \times 10^{10} \mathrm{~Gy}$, respectively. It gives $\sim 3$ year lifetime for copper and ceramic. Annual neutron flux $(\mathrm{E}>0.1 \mathrm{MeV})$ and dose in the hottest spot of the SC coil are $8 \times 10^{17} \mathrm{~cm}^{-2}$ and $1.3 \times 10^{7} \mathrm{~Gy}$, or 15 to 20 year lifetime. The lifetime numbers are somewhat uncertain because of lack of data on radiation damage to SC materials at $\mathrm{E}>14 \mathrm{MeV}$.
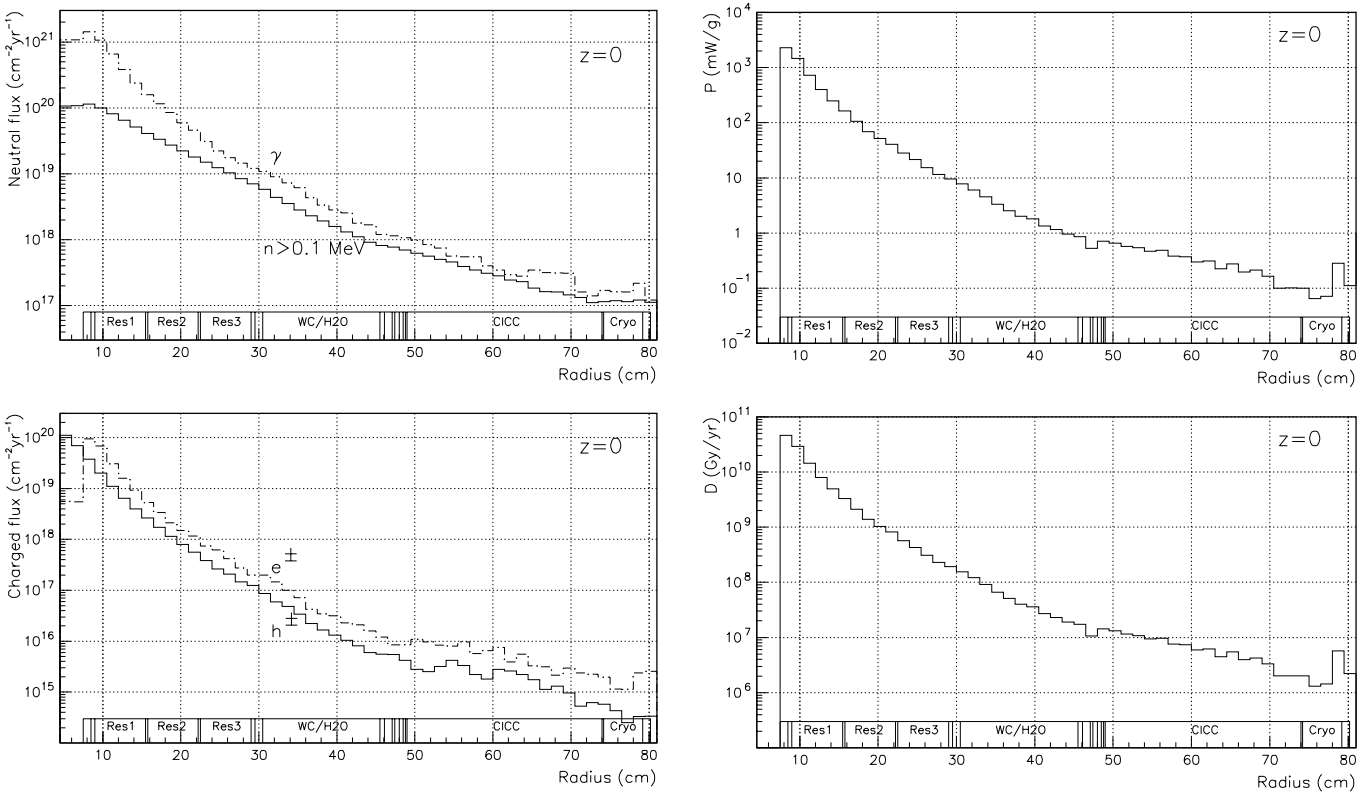

Fig. 3. Radial distribution of particle flux $\left(\mathrm{cm}^{-2} \mathrm{yr}^{-1}\right)$, power density $(\mathrm{mW} / \mathrm{g})$ and absorbed dose (Gy/yr) for a $1.5 \mathrm{MW} 16 \mathrm{GeV}$ beam on a $\mathrm{C}$ target.

\section{Residual dose and shielding}

Fig. 4 (right) shows residual dose rate $P_{\gamma}$ isocontours in the entire system. The rates are up to $10^{7} \mathrm{mSv} / \mathrm{hr}=10^{6} \mathrm{R} / \mathrm{hr}$ on the target, bore tube and inner resistive coil, $10^{3} \mathrm{mSv} / \mathrm{hr}=100 \mathrm{R} / \mathrm{hr}$ on $\mathrm{CICC} \mathrm{SC}$ coil and $10^{2} \mathrm{mSv} / \mathrm{hr}=$ $10 \mathrm{R} / \mathrm{hr}$ on the vessel, which requires remote control and robotics. Radiation shielding needed is $\sim 2 \mathrm{~m}$ of steel followed by $0.3 \mathrm{~m}$ concrete wall/floor to protect ground water followed by about $3 \mathrm{~m}$ of dirt to provide personnel protection. 

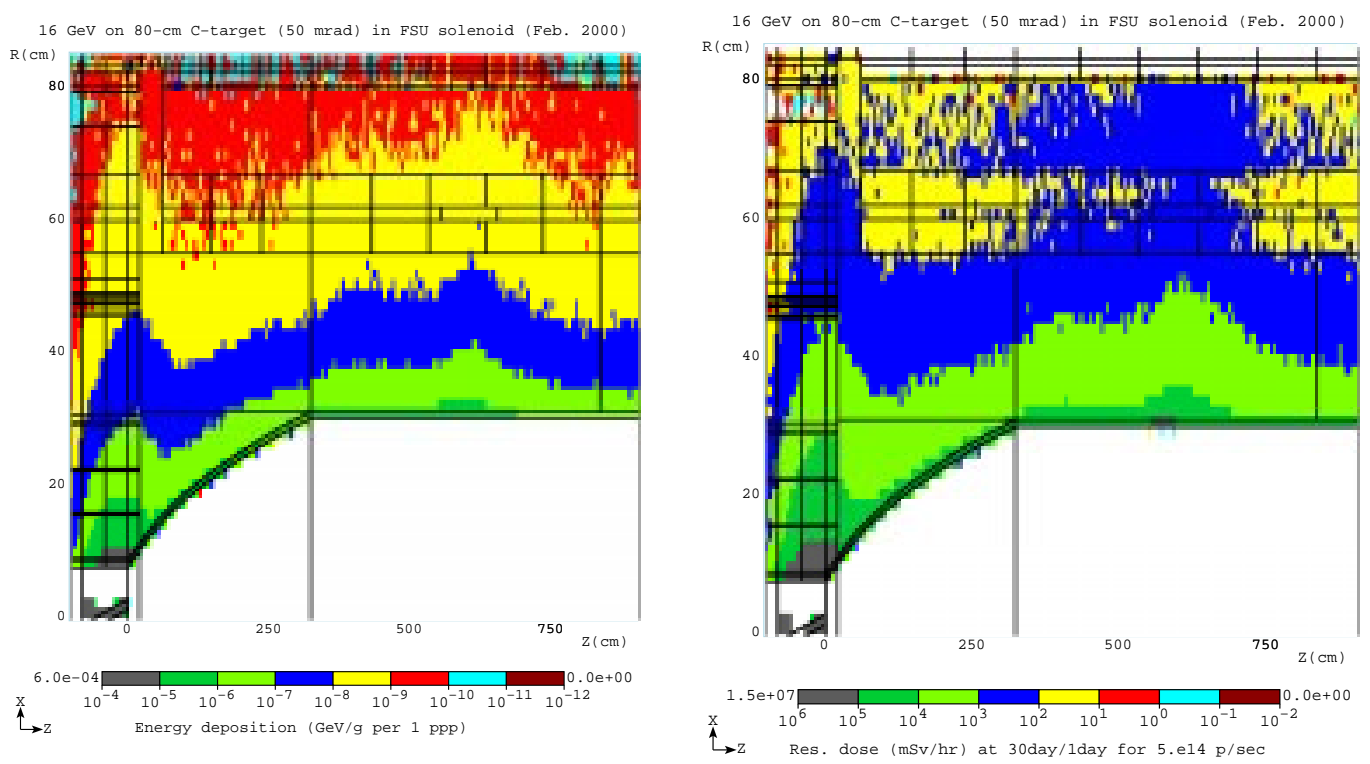

Fig. 4. Energy deposition in $\mathrm{GeV} / \mathrm{g}$ per $1 \mathrm{ppp}$ (left) and residual dose rate after 30 day irradiation and 1 day cooling at $5 \times 10^{14} \mathrm{p} / \mathrm{sec}$ (right).

\section{Primary beam dump}

With a tilted target, a non-interacted part of the proton beam, as well as elastically scattered protons and energetic secondary particles generated in a very forward direction, hit a low-field solenoid aperture at about $6 \mathrm{~m}$ downstream of the target. There is obviously a strong azimuthal asymmetry in flux and dose distributions at this location, as shown in Fig. 5. The annual neutron flux $(\mathrm{E}>0.1 \mathrm{MeV})$ and dose in the hottest spot of the potted SC coil at the beam dump are $7.6 \times 10^{17} \mathrm{~cm}^{-2}$ and $4.1 \times 10^{7} \mathrm{~Gy}$, respectively, or 7-10 year lifetime with the current shielding. Fig. 6 shows azimuthally averaged particle fluxes, power density and accumulated dose. At the longitudinal maximum, these values are 2-2.5 times lower than the azimuthal peaks.
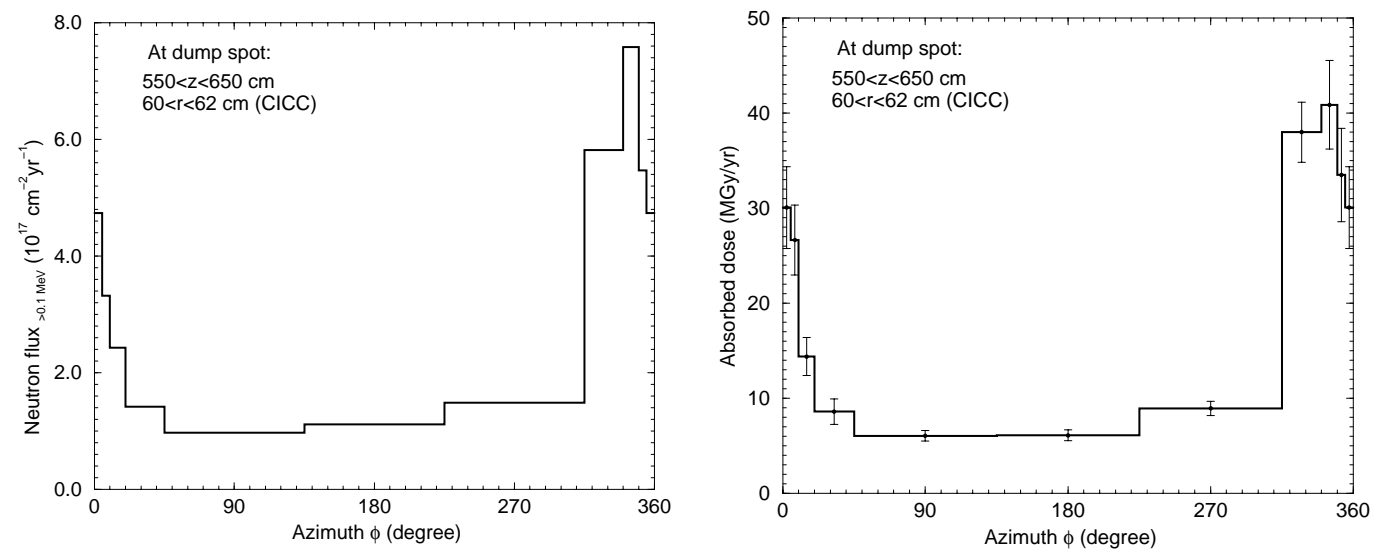

Fig. 5. Azimuthal distribution of neutron $(\mathrm{E}>0.1 \mathrm{MeV})$ flux and absorbed dose in a $\mathrm{SC}$ coil at the dump location for a $1.5 \mathrm{MW} 16 \mathrm{GeV}$ beam. 

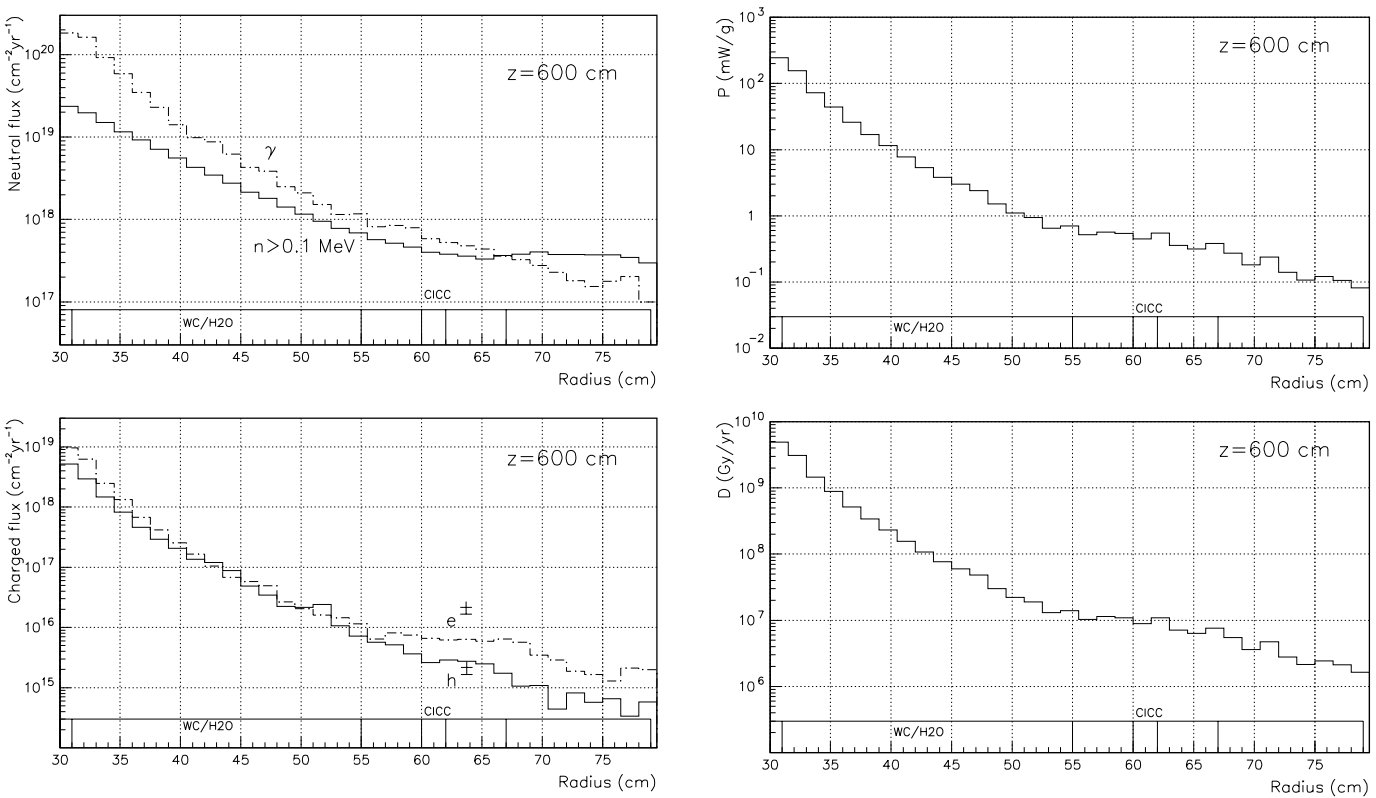

Fig. 6. Azimuthally averaged particle flux $\left(\mathrm{cm}^{-2} \mathrm{yr}^{-1}\right)$, power density $(\mathrm{mW} / \mathrm{g})$ and absorbed dose (Gy/yr) at the dump vs radial distance from the axis.

\section{Conclusions}

The radiation loads and residual dose rates on the innermost target/capture system components are very high. The proposed tungsten-carbide-water shielding in front of the SC coils reduces the operational and accumulated dose in the high-field and low-field coils to quite acceptable levels.

\section{References}

[1] In: N. Holtkamp and D. Finley, eds., A Feasibility Study of a Neutrino Source Based on a Muon Storage Ring, Fermilab-Pub-00/108-E (2000).

[2] N.V. Mokhov, The MARS Code System User's Guide, Fermilab-FN-628 (1995); N.V. Mokhov et al., MARS Code Developments, Fermilab-Conf-98/379 (1998); http://www-ap.fnal.gov/MARS/.

[3] N.V. Mokhov, MARS Code Developments, Benchmarking and Applications, in: Proc. of ICRS-9 International Conference on Radiation Shielding (Tsukuba, Ibaraki, Japan, 1999), J. Nucl. Sci. Tech. 1 (2000) 167-171; Fermilab-Conf00/066 (2000).

[4] N. V. Mokhov, $\pi / \mu$ Yield and Power Dissipation for Carbon and Mercury Targets in 20-Tesla Solenoid with Matching Section, Mucool Note-MUC0061 (1999), http://www-ap.fnal.gov/ mokhov/mumu/target99/muc0061 and updates there. 